\title{
ELECTRONIC FUSE USING RESETTABLE POLY FUSE FOR OVER CURRENT PROTECTION
}

\author{
Shivam Junghare \\ Department of EnTC \\ MVP's KBTCOE, Nashik, Maharashtra, India
}

\begin{abstract}
This paper presents a new technique to deal with the high load currents or fluctuating currents using poly fuse as its major component. Poly fuse is a resettable fuse which goes on increasing its resistance, blocking the excess current until the current is back to normal. Once the current is under normal conditions, the resistance stabilizes and the path between supply and load is reconnected. The proposed work also uses a reed relay which breaks the path exactly at the specified rating, allowing a fast switch. The combination of poly fuse and reed relay provides more efficient outputs as compared to traditional circuit breakers. The fast-switching action, accurate results and the smaller size makes poly fuse more suitable for most of the power devices.
\end{abstract}

Keywords - poly fuse, electronic fuse, over current, circuit brakers, resettable.

\section{INTRODUCTION}

Most of the power devices and electronic circuits around the world uses traditional circuit brakers(fuse) to tackle the over current problem. However, these traditional tin wire fuses have some snags associated with them. Those include:

- The tin wires do not allow the breakdown exactly at the specified rating.

- Some amount of high current passes through them, damaging the circuit.

- After every breakdown, we have to replace the tin wire or rewound it.

To overcome these drawbacks, a device with high efficiency and accuracy was needed. Also, the problem to rewound the tin wire should be eradicated. Something which can break itself by the application of high current and can restore it after the high current stabilizes.

Poly fuses are well known for this kind of operation. With the proper arrangement of poly fuse and relays, a circuit is designed which can actually break the flow of high current at the specified rating. Also, allowing the flow as soon as the current stabilizes. The rest of the paper is organized as follows. Proposed work is explained in section II.
Experimental results are presented in section III. Concluding remarks are given in section IV.

\section{PROPOSED WORK}

A. Reed Relays -
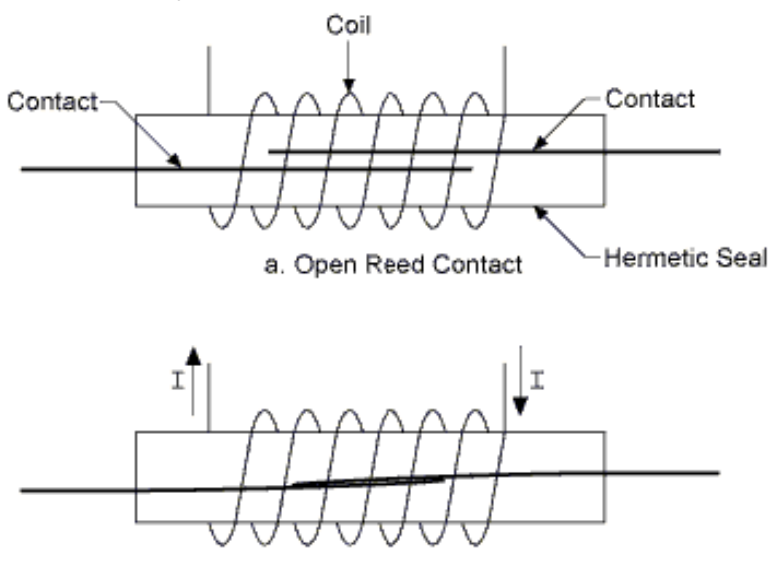

b. Closed Reed Contact

Fig. 1. Working of Reed Relay

The turns of the reed relay winding depend on the current flowing through them. We can set the current rating at which the contacts will close by changing the no. of turns [4].

As the cuurent flows through the coil, magnectic induction causes the contacts to twig, allowing the current to flow through them.

Table 1. Standard ratings

\begin{tabular}{|l|l|l|}
\hline Current (A) & No. of turns & SWG \\
\hline 5 & 10 & 16 \\
\hline 2.5 & 20 & 18 \\
\hline 1.25 & 4 & 21 \\
\hline
\end{tabular}

Table 1 shows the standard current ratings which can be obtained by using the specific SWG and the no. of turns [3]. 
For the requires experiment, we have selected 1A current with 21 SWG, 3.3 turns.

\section{B. Polyfuse -}

A poly fuse is a device which can reset itself after it is broken. Also called as multi fuse, a poly fuse in its broken state, increases resistance, thereby blocking the current to flow. As soon as the excess current is back to normal, the resistance decreases and the path again establishes to let the current flow [1].

Commercially, a variety of poly fuses are available, ranging from $40 \mathrm{~mA}$ to $10 \mathrm{~A}$.

\section{Circuit -}

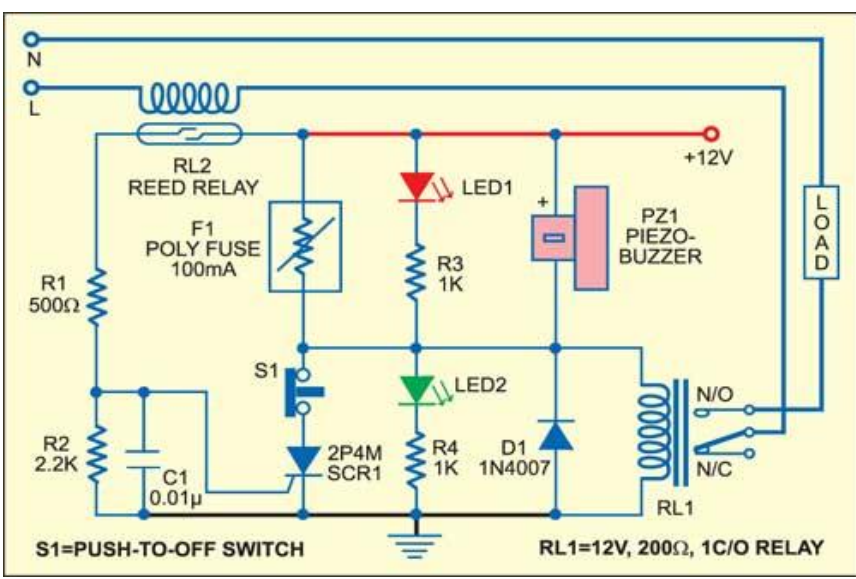

Fig. 2. Complete circuit

\section{EXPERIMENT AND RESULT}

The experiment was performed on Proteus 7 software according to the circuit diagram shown. Input supply is taken as $230 \mathrm{~V} \mathrm{AC,} \mathrm{1A.} \mathrm{For} \mathrm{this} \mathrm{purpose} \mathrm{the} \mathrm{reed} \mathrm{relay} \mathrm{selsected} \mathrm{was}$ of $1 \mathrm{~A}$ with 3.3 turns of 21 SWG.

This supply is given to the load which is connected to a normal $12 \mathrm{~V}$ relay.

The normal relay (RL1) is energised by an internal battery of $12 \mathrm{~V}$. This battery provides a current of $60 \mathrm{~mA}$. Therefore, the poly fuse selected is of rating $60 \mathrm{~mA}$.

When the input supply is $<1 \mathrm{~A}$, the contacts of reed relay are open. The battery energises the relay RL1 and the load receives the power. As soon as the input current is $>=1 \mathrm{~A}$, the reed relay coil energises and the contacts connect. As a result, (the current flows through the lowest resistance path and) the SCR gets triggered and the poly fuse goes in high resistance state, disconnecting the load. A red led and BUZZER gets activated, indicating the failure of the circuit.
Once, the current is back to normal $(<1 \mathrm{~A})$, the reed relay coil deenergises. The circuit will still be in the failure state as SCR will still be in conduction mode. The circuit can be brought back to normal state by pressing thr switch button which is normally pressed. As the contact between poly fuse and SCR breaks, the SCR is brought back to forward blocking mode. Eventually, the path of the current is again from $12 \mathrm{~V}$ polyfuse-green led, RL1-ground. This green led indicates that the current is stable.

Table -2 Experiment Result

\begin{tabular}{|c|c|c|}
\hline Input & $\begin{array}{c}\text { Expected } \\
\text { breakdown at }\end{array}$ & $\begin{array}{c}\text { Actual breakdown } \\
\text { occurred at }\end{array}$ \\
\hline $230 \mathrm{~V}, 1 \mathrm{~A}$ & $1 \mathrm{~A}$ & $\mathbf{0 . 9 9 9 A}$ \\
\hline $230 \mathrm{~V}, 1.2 \mathrm{~A}$ & $1 \mathrm{~A}$ & $1.002 \mathrm{~A}$ \\
\hline $230 \mathrm{~V}, 1.5 \mathrm{~A}$ & $1 \mathrm{~A}$ & $1.004 \mathrm{~A}$ \\
\hline
\end{tabular}

Table 2 shows the experimental results. It compares the expected and actually observed readings. The readings clearly indicates that there is not much difference between the expected and observed readings. With an average lag of just $2 \mathrm{~mA}$.

\section{CONCLUSION}

This paper illustrates a new concept of circuit brakers. The circuit uses poly fuse which eradicates the need of replacing the fault wire as in the case of traditional circuit breakers. Also, the use of reed relays as the switching element, fastens the circuit, while breaking the flow of high current exactly at the specified rating. Overall, the efficiency of the circuit increases by a considerable amount, saving the time required to replace the tin wire. Also, the results performed at $230 \mathrm{~V}, 1 \mathrm{~A}$ are very constructive with only $0.002 \%$ error.

\section{REFERENCE}

[1] Cheng.S, Tom.K, Pecht. M. "Failure precursors for polymer resettable fuses," IEEE transaction on device and material reliability, vol. 10, pp. 374-380, 2010.

[2] Plesca.A, Dumitrescu. C, Zhang.G, Han.D . "Over current protection using a new type of electric fus," International Conference and Exposition on Electrical and Power Engineering, Romania, 2016

[3] Babu.T.A . "Electronic Fuse," Engineering Projects For You, Dec, 2017

[4] Oramus.P, Koska.K, Kozak.J, Chmielevski.T. "Research on Surge Voltage Withstand of Medium Voltage Vaccum Reed Relay," Selected Isuues of Electrical Engineering and Electronics, Szczecin, Poland, 2018 